\title{
Les maladies infectieuses, les déterminants sociaux et la nécessité d'une action intersectorielle
}

\author{
Butler-Jones $D^{1 *}$, Wong $T^{1}$
}

\section{Résumé}

Une lutte efficace contre les maladies infectieuses exige une vaste approche à multiples facettes. Les efforts en matière de santé publique au XIXe siècle ont mis l'accent sur la propreté et les bonnes conditions de vie. La théorie germinale des maladies infectieuses qui a prévalu par la suite a été à l'origine de percées majeures dans le domaine des vaccins et antimicrobiens, mais a également donné lieu à un excès de confiance. Désormais, à la lumière des infections émergentes et ré-émergentes et de la résistance aux antimicrobiens, nous savons qu'une approche unidisciplinaire du contrôle des maladies infectieuses n'est plus suffisante et que c'est en travaillant avec les autres que nous pouvons déterminer des moyens pratiques pour aborder tous les facteurs en jeu dans l'émergence et la persistance des maladies infectieuses. Dans le cadre d'une collaboration intersectorielle, interprofessionnelle ou d'activités intergouvernementales ou de coalition, quatre principes majeurs s'appliquent : le respect, l'aspect pratique, la règle de trois et avoir quelque chose à offrir.

\section{Affiliation}

'Direction générale de la santé des Premières Nations et des Inuits, Santé Canada, Ottawa (Ontario)

${ }^{\star}$ Correspondance : david.butlerjones@hc-sc.gc.ca

Citation proposée : Butler-Jones D, Wong T. Les maladies infectieuses, les déterminants sociaux et la nécessité d'une action intersectorielle. Relevé des maladies transmissibles au Canada 2016;42-Suppl 1:S21-4. https://doi.org/10.14745/ccdr.v42is1a04f

\section{Introduction}

Aborder le défi des maladies infectieuses a toujours nécessité l'adoption d'une vaste approche à multiples facettes. Notre compréhension ayant évolué, cette approche a été élargie de manière à inclure le concept de déterminants sociaux de la santé en vue de mieux aborder l'interaction complexe entre les conditions dans lesquelles nous vivons et notre capacité à favoriser la santé et à nous remettre de la maladie (1). Le présent document décrit l'historique de notre compréhension des maladies infectieuses, de la théorie germinale des maladies infectieuses aux déterminants de la santé et au-delà, définit le besoin d'une approche intersectorielle et le lien avec les principes qui favorisent la formation de coalitions et l'efficacité des mesures intersectorielles.

\section{Théorie germinale des maladies infectieuses}

La théorie germinale des maladies infectieuses a été à l'origine de percées majeures qui ont contribué à l'éradication de certaines maladies et à la quasi-élimination d'autres maladies grâce à l'utilisation des vaccins et des antimicrobiens. Quoi qu'il en soit, cette théorie a été très controversée et a fait l'objet de nombreuses discussions au XIX $\mathrm{X}^{\mathrm{e}}$ siècle. II y avait, par exemple, ceux qui craignaient qu'en concluant que les germes étaient la cause réelle de la maladie, les gens ne chercheraient plus à assainir les conditions d'insalubrité souvent à l'origine des infections (2).

La confiance démesurée qui a caractérisé le milieu du $X X^{e}$ siècle à amener à penser que l'infection ne constituait plus une grave menace pour l'humanité (3). La théorie germinale des maladies infectieuses ayant été adoptée à grande échelle la tendance à faire preuve d'un peu plus de complaisance avec les conditions environnantes est devenue un problème. Par exemple, au cours des interventions chirurgicales, les personnes considéraient que les antibiotiques pouvaient régler tout risque d'infections postopératoires et se sont montrées davantage laxistes à l'égard des techniques aseptiques. De manière générale, moins d'attention a été accordée au contrôle des infections et aux efforts en matière de santé publique.

La complaisance en matière de maladies infectieuses est aujourd'hui pratiquement absente. Ce changement a commencé dans les années 1980 et 1990, avec l'apparition de l'épidémie de $\mathrm{VIH}$, puis s'est poursuivi au cours des années 2000, avec le syndrome respiratoire aigu sévère et la grippe pandémique (H1N1). L'émergence de la résistance aux antimicrobiens (p. ex. tuberculose multirésistante), I'apparition de nouvelles maladies infectieuses (p. ex. le virus Ebola) et la réapparition d'anciennes maladies infectieuses que l'on croyait vaincues (p. ex. la coqueluche) ont également remis en question la complaisance.

Plus nous en savons au sujet de l'infection, plus nous nous rendons compte de tout ce que nous devons apprendre. Les prions et les maladies liées à la propagation de protéines révèlent un type de maladie infectieuse qui n'est pas causée par un micro-organisme, et il ne s'agira probablement pas de la

dernière forme d'organisme infectieux que nous allons découvrir (4). Chaque année, de nouvelles connaissances nous permettent de mieux comprendre la façon dont les micro-organismes 
causent ou favorisent un éventail croissant de maladies et de troubles immunitaires.

\section{L'interaction entre les microbes et les déterminants sociaux}

Nous avons aujourd'hui reconnu, ou peut-être redécouvert, I'influence significative des déterminants sociaux sur les taux de maladies infectieuses et non infectieuses, et sur la mortalité. L'endroit où nous vivons et la façon dont nous nous comportons, notre contexte social, environnemental et économique, influent tous sur notre bien-être et notre survie.

Nous avons, en quelque sorte, bouclé la boucle. Nous nous sommes rendus compte que la théorie germinale des maladies infectieuses était un peu trop simple et nous avons acquis aujourd'hui une compréhension plus nuancée du rôle que les micro-organismes jouent dans certaines maladies, de leur fonction dans la promotion de processus physiologiques normaux, comme la digestion, et de leurs interactions complexes avec l'immunité (5). Cependant, nous avons également constaté qu'il y avait beaucoup de substance au début du mouvement de santé publique, dont la priorité était une meilleure hygiène et l'amélioration générale des conditions économiques et sociales. Bien que, dans l'ensemble, les conditions sociales et économiques de la santé se sont beaucoup améliorées au cours du dernier siècle, en particulier dans les pays développés, la négligence des questions relatives aux déterminants sociaux a généré des inégalités $(6,7)$. Pour aider à prévenir et à traiter les maladies et les blessures, nous devons tenir compte de toutes les connaissances que nous avons sur les micro-organismes, les vaccins, les traitements et les déterminants sociaux de la santé. Cela étant, le concept « Une santé » est de plus en plus accepté comme une autre façon d'envisager les problèmes auxquels nous sommes confrontés. C'est dans l'interaction entre les animaux, les humains, les facteurs environnementaux et économiques que se trouvent de nombreuses solutions aux défis toujours plus complexes que nous devons affronter.

Un exemple fascinant de l'interaction entre les déterminants sociaux de la santé et les microbes est le fait que l'incidence de la tuberculose a commencé à décliner dans une grande partie de la société occidentale avant l'arrivée de traitements médicaux efficaces (8). De meilleurs logements, moins de surpeuplement, I'amélioration de la nutrition et des conditions de vie, ainsi que la pasteurisation, parmi d'autres facteurs, ont contribué au déclin marqué du taux de tuberculose au $X I X^{e}$ siècle et au début du $X X^{e}$ siècle en Europe et en Amérique du Nord (9).

Ironiquement, bien qu'un certain nombre de traitements efficaces pour la tuberculose ont été développés dans la seconde moitié du XXe siècle, la maladie n'a pas été éliminée. Cela s'explique par une combinaison des déterminants sociaux de la santé et de facteurs immunologique (tels que l'augmentation de la tuberculose chez les personnes atteintes du $\mathrm{VIH})$, et l'émergence de souches de la bactérie multirésistante (10). La tuberculose demeure endémique dans de nombreux pays, y compris dans les Amériques, chez les populations autochtones. L'une des clés de la solution à plus long terme pour lutter contre la tuberculose dans le nord du Canada est l'accès à un logement adéquat en plus de la prévention et du traitement (11-13).
La tuberculose n'est pas le seul exemple d'interaction importante entre les microbes et les déterminants sociaux de la santé. Le fardeau de Staphylococcus aureus résistant à la méthicilline (SARM) et le virus respiratoire syncytial (RSV) est associée à un logement inadéquat et à la pauvreté $(14,15)$. Même le fardeau du $\mathrm{VIH} /$ sida (qui sont les personnes touchées et comment elles réagissent au traitement) est lié aux déterminants sociaux de la santé. Par exemple, les personnes vivant avec le $\mathrm{VIH}$ qui ont un logement stable sont plus susceptibles d'avoir une meilleure observance du traitement (16).

Pour lutter efficacement contre les maladies infectieuses, la question n'est plus de savoir si l'accent doit être mis sur le soutien social ou sur les facteurs médicaux, biologiques ou environnementaux $(17,18)$, tous ces facteurs jouant un rôle important. Cela signifie également qu'une seule personne ou un seul groupe ne peut pas s'attaquer à toutes les maladies infectieuses. En fait, pour pouvoir lutter efficacement contre les maladies infectieuses aujourd'hui, plusieurs compétences sont nécessaires : la connaissance des maladies infectieuses, la santé publique et les déterminants sociaux de la santé, et la capacité à travailler avec les communautés locales, les gouvernements et les organisations non gouvernementales.

\section{Pourquoi nous avons besoin de coalitions et de l'action intersectorielle}

Les stratégies unidiscipinaires de contrôle des maladies ne seront jamais réellement suffisantes pour lutter contre de nombreuses maladies infectieuses : les déterminants sociaux de la santé sous-jacents doivent aussi être pris en compte. Si nous gardons à l'esprit l'importance des déterminants sociaux de la santé et d'autres facteurs non microbiens au moment d'organiser les services de santé, nous trouveront plus facilement des stratégies efficaces.

Un exemple de l'application multidisciplinaire efficace de contrôle des maladies infectieuses est l'utilisation d'un vaccin pour arrêter les éclosions périodiques de l'hépatite A dans les petites communautés éloignées en Saskatchewan. Tout en préconisant une amélioration des conditions sociales et de logement, le système de soins de santé a offert un programme d'immunisation et, par conséquent, a permis de réduire les taux de la maladie au sein de ces communautés à une valeur inférieure à celle de la province dans son ensemble (données non publiées de la Saskatchewan sur les maladies transmissibles de 1994 à 1999, Dr Shauna Hudson).

L'un des défis que nous devons relever pour traiter les déterminants sociaux de la santé est que ceux-ci ne sont pas simplement déterministes. En d'autres termes, ce n'est pas parce que la situation financière d'une personne est bonne ou précaire que sa santé sera bonne ou mauvaise. La pauvreté n'est pas qu'un problème économique; il s'agit d'un ensemble de ressources manquantes, de liens, d'autodétermination, d'environnement, etc. Certaines collectivités s'en sortent beaucoup mieux que d'autres avec des revenus, un environnement, une génétique et une culture de base similaires. De plus ces facteurs peuvent être atténués davantage avec des efforts appropriés dans les domaines de l'éducation, de la santé et des services sociaux. 
Avec une telle diversité dans les conditions humaines et les méthodes pour aborder ces problèmes, l'un de nos principaux défis est d'aider les collectivités à passer de ce qu'elles sont à ce qu'elles pourraient être.

Parfois, les problèmes reliés à la pauvreté, aux facteurs sociaux, environnementaux et économiques, et notre capacité à les influencer semblent écrasants. Bien que personne ne puisse y parvenir seul et que certains défis semblent insurmontables, diviser ces problèmes en plusieurs problèmes abordables peut être utile pour garantir une action positive.

\section{Principes de la collaboration avec divers secteurs et coalitions}

Aucun modèle ou technique idéale ne peut convenir à toutes les personnes ni à toutes les situations. La solution est d'améliorer notre compréhension des différents facteurs sous-jacents des risques et des avantages pour la santé grâce à la collaboration avec d'autres personnes, afin de pouvoir identifier des façons pratiques pour aborder tous les facteurs qui pourraient être en cause.

Les compétences nécessaires pour traiter les maladies infectieuses dans le contexte des déterminants sociaux de la santé peuvent nous sortir de nos rôles habituels. La diplomatie, la formation de coalitions, la création de communautés ne sont que quelques-unes des principales capacités qui nous permettront de gérer les risques et les facteurs sous-jacents. Quatre principes importants s'appliquent.

\section{Respect}

Nous ne pouvons pas influencer ceux ou ce que nous ne respectons pas. Bien qu'il ne soit pas nécessaire de considérer ceux avec lesquels nous travaillons pour atteindre des objectifs communs comme des amis - ni même d'être d'accord avec eux - nous devons les respecter en tant que personnes et comprendre les défis auxquels ils sont confrontés, ainsi que leurs aspirations. Ce n'est qu'alors que la conversation sur les changements potentiels pourra avoir lieu. Lorsque nous nous sentons frustrés par les différences, une harangue peut sembler thérapeutique, mais cela entraîne rarement un changement significatif de perspective; la réaction la plus courante est de souhaiter avoir le moins possible affaire avec les harangueurs. C'est pourquoi il est essentiel de travailler dans un esprit de respect.

\section{Trouver des solutions pratiques}

Identifier les problèmes n'est pas suffisant. Les responsables des politiques, entre autres, ont également besoin de solutions pratiques et faisables qui soient démontrables ou réalisables. Appliquer ce que nous connaissons déjà ou ce que nous pouvons acquérir dans d'autres situations et comprendre comment procéder à une mise en œuvre efficace peut signifier aller de l'avant sans avoir à recourir à de nouvelles stratégies et technologies.

\section{La règle de trois}

Trop souvent, les points de désaccord entre les personnes deviennent les sujets principaux des réunions et entravent les progrès. Pour résoudre ce problème, arrêtez-vous, faites le bilan et divisez les problèmes en trois catégories : ceux sur lesquels on peut s'entendre, ceux qui ne sont pas parfaits mais avec lesquels on peut vivre et ceux qui n'aboutiront probablement pas à un accord. Sans toutefois ignorer complètement cette dernière catégorie, axer la plupart des efforts sur les deux autres empêche qu'une seule de ces catégories n'utilise plus qu'une petite part du temps et de l'énergie disponibles.

\section{Avoir quelque chose à offrir}

Il est rare qu'une seule organisation dispose du mandat ou de la capacité nécessaire pour répondre seule aux grandes questions complexes. La raison de la formation de coalitions est que les différents groupes ont différentes choses à offrir, c'est pourquoi il est très utile d'identifier ce que vous et votre organisation pouvez offrir. Une conversation qui va dans le sens de « nous pouvons faire ceci si vous pouvez faire cela » a constitué une technique utile pour mettre fin à l'accumulation des problèmes entre les organisations.

\section{Conclusion}

L'amélioration de notre approche biomédicale pour lutter contre les maladies infectieuses avec des stratégies efficaces fondées sur les déterminants sociaux de la santé et l'approche Une santé sera un élément clé de la réussite à long terme. Que ce soit en médecine ou en politique, les principes fondamentaux sont importants. II faut veiller à ce que les mesures les moins intrusives et les plus efficaces, avec le moins d'effets secondaires possible, nous soient utiles à tous.

\section{Remerciements}

Nous souhaitons remercier tous ceux qui travaillent dans des coalitions et qui facilitent l'action intersectorielle pour améliorer la santé de tous.

\section{Conflit d'intérêts}

Aucun.

\section{Financement}

Aucun.

\section{Références}

1. Butler-Jones D, editor. The Chief Public Health Officers report on the state of public health in Canada, 2008: addressing health inequalities. Ottawa (ON): Public Health Agency of Canada; 2008. (Disponible en français :http:// www.phac-aspc.gc.ca/cphorsphc-respcacsp/2008/fr-rc/pdf/ CPHO-Report-f.pdf) 
17. Butler-Jones D. Commentary in: Goel V, Mclsaac W. Clinical general practice as a setting for health promotion. In: Poland BD, Green LW, Rootman I, editors. Settings for health promotion: linking theory and practice. Thousand Oaks (CA): Sage; 2000.

18. Heymann J., Hertzman C, Barer ML, Evans RG. Healthier societies: from analysis to action. New York: Oxford University Press; 2006.

5. Butler-Jones D, editor. The Chief Public Health Officer's report on the state of public health $n$ Canada, 2013: infectious disease-the never-ending threat. Ottawa (ON): Public Health Agency of Canada; 2013. (Disponible en français : http://www.phac-aspc.gc.ca/cphorsphcrespcacsp/2014/assets/pdf/2014-fra.pdf).

6. Postl BD, Cook CL, Moffatt M. Aboriginal child health and the social determinants: why are these children so disadvantaged? Healthc Q. 2010;14:42-51.

7. Richmond CR. Ross NA, Egeland GM. Social support and thriving health: a new approach to understanding the health of indigenous Canadians. Am J Public Health. 2007;97(10):1827-33.

8. McKeown T. The role of medicine: dream, mirage or nemesis? Princeton (NJ): Princeton University Press; 1979.

9. Hargreaves JR, Boccia D, Evans CA, Adato M, Petticrew $M$, Porter JD. The social determinants of tuberculosis: from evidence to action. Am J Public Health, 2011;101:654-62.

10. Brudney K, D. J. Resurgent tuberculosis in New York City: Human immunodeficiency, homelessness, and the decline of the tuberculosis program. AM Rev Resp Dis. 1991; 144:745-9.

11. Canadian Tuberculosis Committee. Housing conditions that serve as risk factors for tuberculosis infection and disease. Can Comm Dis Report. 2007;33 (ACS-9). (Disponible en français : http://www.phac-aspc.gc.ca/publicat/ccdrrmtc/07vol33/acs-09/index-fra.php)

12. Clark M, Riben P, Nowgesic $E$. The association of housing density, isolation and tuberculosis in Canadian First Nations communities. Int J Epidemiol. 2002;31(5):940-5.

13. Kulmann KC, Richmond CA. Addressing the persistence of tuberculosis among the Canadian Inuit population: the need for a social determinants of health framework. Int Indigen Policy J. 2011;2(1):1-16.

14. Tong SY, McDonald MI, Holt DC, Currie BJ. M. Global implications of the emergence of community-associated methicillin-resistant Staphylococcus aureus in Indigenous populations. Clin Infect Dis. 2008;46:1871-8.

15. Colosa AD, Masaquel A, Hall CB, Barrett AM, Mahadevia PJ, Yogev R. Residential crowding and severe respiratory syncitial virus disease among infants and young children: a systematic literature review. BMC Infect Dis. 2012;12 (95.doi:10.1186/171-2334-12-95).

16. Rourke SB, Bacon J, McGee F, Gilbert M. Tackling the social and structural drivers of HIV in Canada. Can Comm Dis Rep. 2015;41(12). (Disponible en français: http://www.phac-aspc. gc.ca/publicat/ccdr-rmtc/15vol41/dr-rm41-12/ar-03-fra.php). 